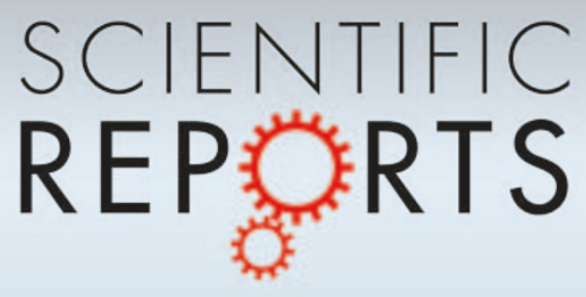

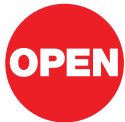

SUBJECT AREAS:

BIOMATERIALS

BIOPHYSICS

PHYSICS

POLYMERS AND SOFT MATERIALS

Received

9 January 2012

Accepted

2 February 2012

Published

22 February 2012

Correspondence and requests for materials should be addressed to

K.Y. (yoshikaw@ scphys.kyoto-u.ac.jp)

\section{Cell-Sized confinement in microspheres accelerates the reaction of gene} expression

\author{
Ayako Kato' , Miho Yanagisawa'2, Yuko T. Sato', Kei Fujiwara3 \& Kenichi Yoshikawa'
}

'Department of Physics, Graduate School of Science, Kyoto University, Kyoto 606-8501, Japan, ${ }^{2}$ Department of Physics, Graduate School of Sciences, Kyushu University, Fukuoka 812-8581, Japan, ${ }^{3}$ Department of Bioengineering and Robotics, Graduate School of Engineering, Tohoku University, Sendai 980-8579, Japan.

Cell-sized water-in-oil droplet covered by a lipid layer was used to understand how lipid membranes affect biochemical systems in living cells. Here, we report a remarkable acceleration of gene expression in a cell-sized water-in-oil droplet entrapping a cell-free translation system to synthesize GFP (green fluorescent protein). The production rate of GFP ( $\left.V_{\mathrm{GFP}}\right)$ in each droplet remained almost constant at least for on the order of a day, which implies $0^{\text {th }}$-order reaction kinetics. Interestingly, $V_{\mathrm{GFP}}$ was inversely proportional to radius of droplets $(R)$ when $R$ is under $50 \mu \mathrm{m}$, and $V_{\mathrm{GFP}}$ in droplets with $R \sim 10 \mu \mathrm{m}$ was more than 10 times higher than that in the bulk. The acceleration rates of GFP production in cell-sized droplets strongly depended on the lipid types. These results demonstrate that the membrane surface has the significant effect to facilitate protein production, especially when the scale of confinement is on the order of cell-size.

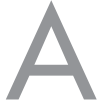

11 living things on Earth maintain their lives by using closed membranes, ranging in size from $\sim 1$ to $\sim 100 \mu \mathrm{m}$. However, it is not yet clear why this scale is so ubiquitous. To gain insight into the effect of cellsized confinement, many studies have been performed on model cellular systems. For example, gene expression has been monitored in liposomes ${ }^{1-10}$. Nomura et al. found that green fluorescent protein (GFP) gene expression is accelerated inside cell-sized giant liposomes $(\sim 5 \mu \mathrm{m})$, by using giant liposomes obtained by natural swelling ${ }^{1}$. Despite this interesting observation, it has been rather difficult to evaluate the confinement effect in a quantitative manner, due to the technical difficulties of encapsulating desired amounts of proteins, DNA and substrates within a size-controlled confinement space. As a related phenomenon, GFP gene expression is known to be promoted in the presence of small phospholipid liposomes in the bulk solution ${ }^{11}$. Thus, it would be worthwhile to clarify the possible acceleration of protein production in a cell-sized confined space covered by a phospholipid layer.

Recently, cell-sized water-in-oil (W/O) droplets coated with a lipid layer have been shown as a useful system for analyzing the interaction between a lipid membrane and encapsulating bio-macromolecules ${ }^{12-16}$. It is easy to control the size of such a droplet system from 1 to $200 \mu \mathrm{m}$, and the system is quite stable independent of the concentration of the entrapped solution and the type of lipid used, which is problematic when using a closed vesicle or liposome ${ }^{17}$. Furthermore, the droplet can be continuously monitored from soon after encapsulation for more than a day without evaporation ${ }^{18}$. These features enable us to analyze the size effect, lipid-dependence, and time development. In fact, Fiordemondo and Stano indicated that GFP gene expression was enhanced in small droplets coated by lecithin ${ }^{19}$. Although this report is very interesting, the size dependence on the acceleration of GFP gene expression and its lipid-dependence have not been studied in a systematic manner.

In this study, we encapsulated a GFP gene expression system in cell-sized W/O droplets of different sizes $(10-100 \mu \mathrm{m})$ and monitored the size-dependence of GFP expression based on the fluorescent intensity per unit volume using a confocal fluorescence microscope. Our results suggest that confinement within a volume that is on the size order of living cells is favorable for the production of protein, i.e., gene expression.

\section{Results}

Figure 1A shows confocal images of DOPG droplets entrapping a GFP expression system at $3 \mathrm{~h}$ after encapsulation. The fluorescent image (Figure 1A, left) shows the distribution of GFP fluorescence. The merged image (Figure 1A, right) shows that GFP fluorescence was homogeneously distributed in each droplet. Interestingly, the intensity of GFP fluorescence in droplets was strongly dependent on the droplet size. We calibrated the GFP 
(A)

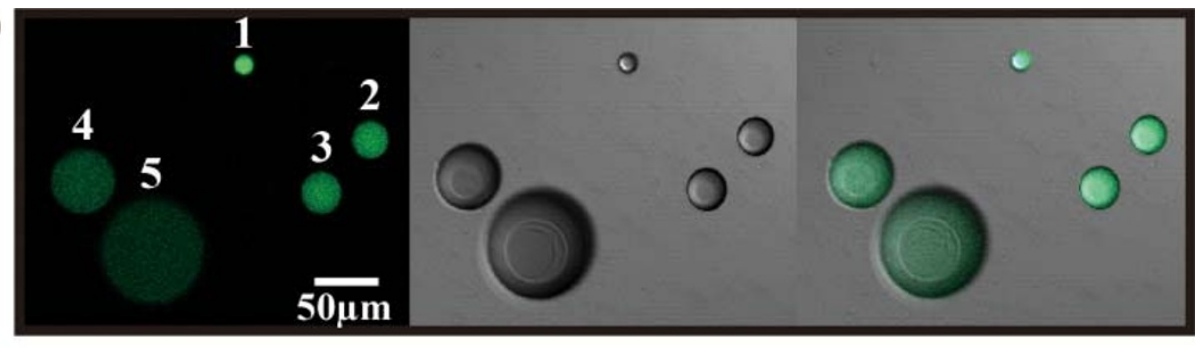

(B)

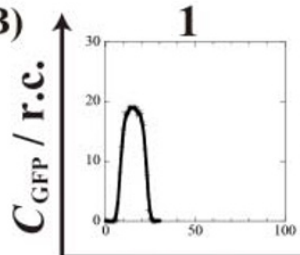

2

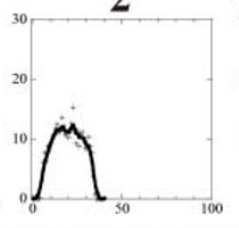

3

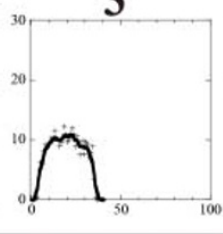

4

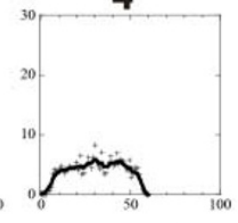

5

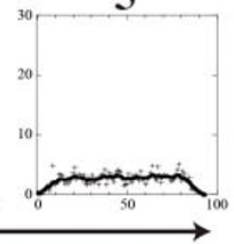

Droplet size, $2 R / \mu \mathrm{m}$

Figure $1 \mid$ (A) Distribution of GFP fluorescence in DOPG droplets with different sizes, at $3 \mathrm{~h}$ after encapsulation. These cross-sectional images show (left) GFP fluorescence, (center) the oil/water interface, and (right) the merged image. The radius of the droplets $R$ is $(1) \sim 10,(2) \sim 15,(3) \sim 15,(4) \sim 25$, and (5) $\sim 45 \mu \mathrm{m}$, respectively. (B) Profiles of the GFP concentration, $C_{\mathrm{GFP}}$, along the diameter, where $C_{\mathrm{GFP}}$ was evaluated from the GFP fluorescence intensity per unit volume normalized by the value of bulk solution at $3 \mathrm{~h}$. The r. c. stands for relative concentration. Apparent sizes of the droplets are somewhat larger $(\sim 10 \mu \mathrm{m})$ owe to the blurring effect in the fluorescent images.

intensity per volume and obtained the GFP concentration $C_{\mathrm{GFP}}$, based on normalization by the value for the bulk solution at $3 \mathrm{~h}$. As shown in Figure $1 \mathrm{~B}, C_{\mathrm{GFP}}$ decreased with an increase in the droplet size. In the case of a small droplet with radius $R \sim 10 \mu \mathrm{m}$ (Figure $1 \mathrm{~B}, 1$ ), the value of $C_{\mathrm{GFP}}$ was $\sim 4$ times larger than that for a large droplet with a radius $\sim 45 \mu \mathrm{m}$ (Figure $1 \mathrm{~B}, 5$ ). On the other hand, $C_{\mathrm{GFP}}$ values in droplets with almost the same $R \sim 15 \mu \mathrm{m}$ were similar (Figure $1 \mathrm{~B}, 2,3$ ). We have confirmed that there was no sizedependence of $C_{\mathrm{GFP}}$ among droplets under the encapsulation of GFP already expressed in the bulk solution (Supplemental Figure S1). Furthermore, this result indicates that the membrane surface and/or total volume significantly affects GFP expression, since the surface per volume ratio is greater with a smaller droplet. If the lipid membrane contributed to the size-dependence of GFP expression, the lipid species used should affect the relation between the droplet size and expression level. Thus, in the following experiments, we examined the effect of different lipids on the confinement effect.

Three different species of lipids, anionic DOPG and zwitterionic DOPC and DOPE, were adapted to encapsulate the GFP gene expression system within droplets. The time-courses of GFP concentration per unit volume, $C_{\mathrm{GFP}}$, were monitored for $\sim 45 \mathrm{~h}$ beginning soon after encapsulation. Identical droplets with a radius $R=$ $10-100 \mu \mathrm{m}$ were monitored (Supplemental Figure S2). As shown in Figure 2, the value of $C_{\mathrm{GFP}}$ linearly increased with time up to $\sim 20 \mathrm{~h}$, and reached a saturation point at $\sim 40 \mathrm{~h}$. Among all three types of lipids, $C_{\mathrm{GFP}}$ in a small droplet with $R \sim 20 \mu \mathrm{m}$ was higher than that in a large droplet with $R>50 \mu \mathrm{m}$. In addition, $C_{\mathrm{GFP}}$ at a certain time and the production rate $V_{\mathrm{GFP}}\left(=d C_{\mathrm{GFP}} / d t\right)$ were both dependent on the type of lipid.

The time-courses of GFP expression levels in the bulk solution were also monitored by encapsulating the solution in PC droplets at each time point (black lines in Figure 2, Supplemental Figure S1). To avoid effects due to the membrane surface, $C_{\mathrm{GFP}}$ was calibrated within $\sim 30$ min after the encapsulation. In contrast to the expression inside droplets, GFP expression levels in the bulk at $25 \mathrm{~h}$ were quite low. For a PG droplet with $R \sim 27 \mu \mathrm{m}, C_{\mathrm{GFP}}$ was more than 15 times higher than that in the bulk (Figure $2 \mathrm{~A}$ ). The rate of acceleration, $V_{\mathrm{GFP}}$, in the bulk at $<20 \mathrm{~h}$ was much less than that in droplets. These results suggest that a lipid membrane facilitates effective gene expression.
To clarify the accelerating effect of the membrane surface, $C_{\mathrm{GFP}}$ at $25 \mathrm{~h}$ was plotted against $1 / R$ in Figure $3 \mathrm{~A}$. The data are fitted by the equation $C_{\mathrm{GFP}}=C_{\mathrm{v}}+C_{\mathrm{s}}(1 / R)$, where $C_{\mathrm{v}}$ and $C_{\mathrm{s}}$ are variable constants related to the inner volume and membrane surface, respectively. Consequently, a linear correlation between $C_{\mathrm{GFP}}$ and $1 / R$ was observed with each lipid, unlike with the bulk solution (black line in Figure 3A). The slopes of the fitted line, $C_{s}$, were different among the three types of lipids, and were approximately 1870 for PG, 1220 for PC, 370 for PE (i.e., PG $>$ PC $>$ PE). On the other hand, the intercept coefficients of the fitted lines in each lipid, $C_{\mathrm{v}}$, were almost the same at $\sim 1$, which means that $C_{\mathrm{GFP}}$ in a large droplet with $R \gg 100 \mu \mathrm{m}$ is closer to that in the bulk solution. These results clearly showed that the cell-sized confinement space surrounding the membrane surface accelerates GFP gene expression.

Finally, the GFP production rate, $V_{\mathrm{GFP}}\left(=d C_{\mathrm{GFP}} / d t\right)$, was plotted against $1 / R$ in Figure $3 \mathrm{~B}$, where, the values of $V_{\mathrm{GFP}}$ were deduced from the slopes of GFP expression levels per unit time up to $\sim 20 \mathrm{~h}$ (Figure 2). Figure $3 \mathrm{~B}$ indicated that $V_{\mathrm{GFP}}$ was linearly correlated with $1 / R$, i.e., $V_{\mathrm{GFP}}=V_{\mathrm{v}}+V_{\mathrm{s}}(1 / R)$, where $V_{\mathrm{v}}$ and $V_{\mathrm{s}}$ are the kinetics from the contributions of the volume and membrane surface, respectively. As a result, $V_{\mathrm{s}}$ was dependent on the lipid species in the order PG $>$ $\mathrm{PC}>\mathrm{PE}$, whereas $V_{\mathrm{v}}$ was quite similar to that in the bulk $\left(\sim 0.02 \mathrm{~h}^{-1}\right)$. This result also suggests that the membrane surface strongly affects gene expression.

Taken together, our quantitative analysis clearly shows that the membrane surface in a cell-sized system accelerates GFP gene expression. It should be noted that this acceleration was not attributed from oxygen solved in oil, because the oil was held under an atmosphere of nitrogen gas before and during the preparation.

\section{Discussion}

Based on the experimental results shown in Figure 3, the GFP concentration per unit volume, $C_{\mathrm{GFP}}$, and the production rate, $V_{\mathrm{GFP}}$, are both proportional to the reciprocal of the droplet size, $1 / R$. Thus, the reaction kinetics is characterized as $0^{\text {th }}$-order, being confirmed from the fact that $V_{\mathrm{GFP}}$ remains almost constant for at least on the order of a day (Figure 2). $0^{\text {th }}$-order is a typical kinetics for the enzymatic reactions. As the next, we discuss the contributions of the kinetics from the bulk and surface. We denote $N$ as a total amount of the enzymatic molecules, which catalyze the production of GFP under a 
(A)

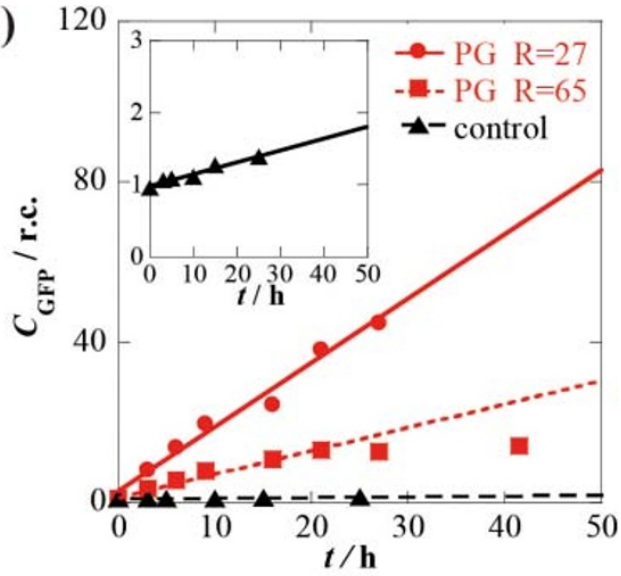

(B)

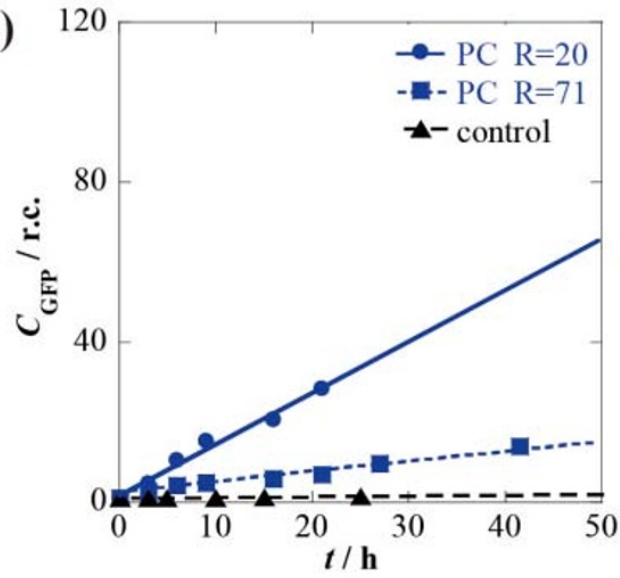

(C)

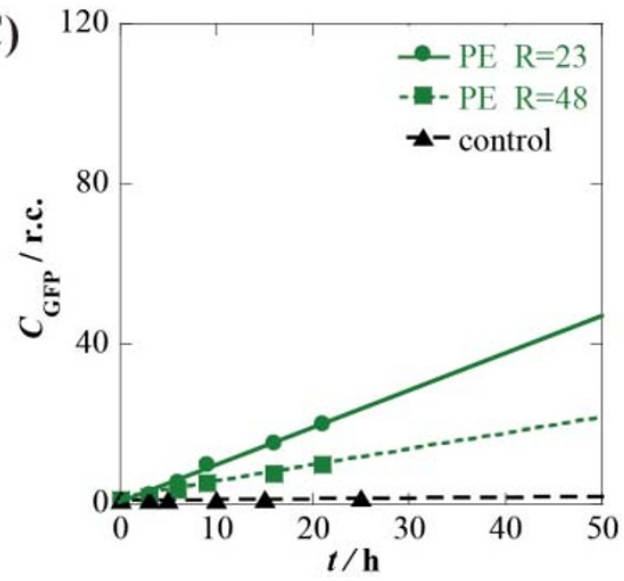

Figure $2 \mid$ Time-courses of the GFP concentration per unit volume, $C_{\mathrm{GFP}}$, in small (radius $R \sim 20 \mu \mathrm{m}$; circle) and large $(R>\sim 50 \mu \mathrm{m}$; square) droplets coated by a lipid layer of (A) DOPG, (B) DOPC, or (C) DOPE, respectively. $C_{\mathrm{GFP}}$ of the bulk solution is shown as a control (triangle). These data fit linear lines from 0 to $20 \mathrm{~h}$, and $C_{\mathrm{GFP}}$ at $3 \mathrm{~h}$ in the bulk solution is taken as unity for all of the graphs.

constant speed in the presence of excess amount of substrates. To gain the essential insight on the reaction kinetics, we discuss the mechanism under a simple framework, i.e., the enzyme is a single species working under a large amount of substrates, i.e., $N \sim$ $C_{\mathrm{GFP}} \cdot 4 \pi R^{3} / 3$. When $N_{0}$ enzyme molecules are encapsulated in a spherical droplet with a radius $R$, they are partitioned into two groups related to the inner volume $N_{\mathrm{v}}$ and membrane surface $N_{\mathrm{s}}$, as follows,

$$
N_{0}=N_{\mathrm{v}}+N_{\mathrm{s}} \text {, and }
$$

(A)

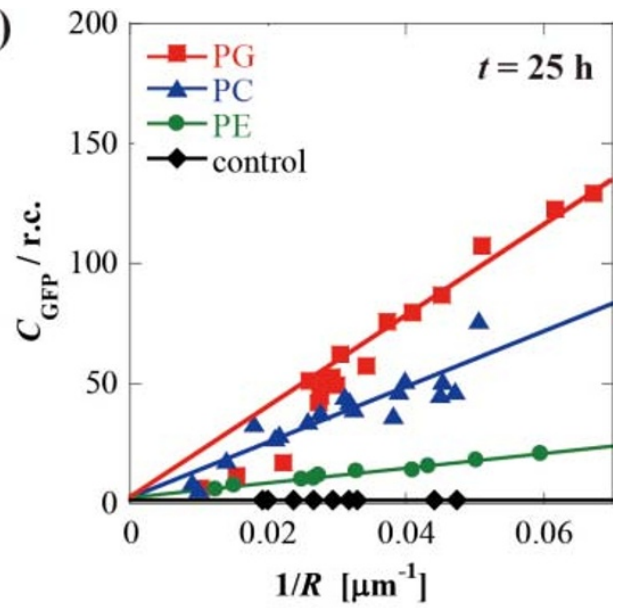

(B)

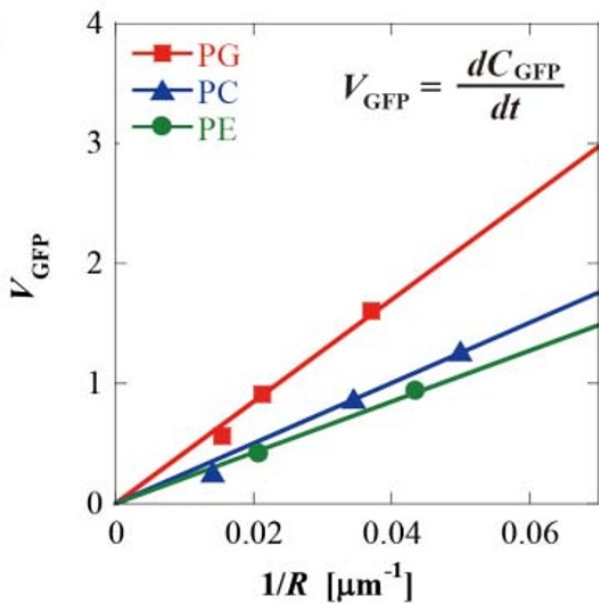

Figure $3 \mid$ The size-dependence of (A) the GFP concentration per unit volume $\mathrm{C}_{\mathrm{GFP}}$ at $25 \mathrm{~h}$, and (B) the production rate $V_{\mathrm{GFP}}$ from 0 to $20 \mathrm{~h}$, evaluated from the slope of linear increase on $\mathrm{C}_{\mathrm{GFP}}$. The lipids are DOPG (square), DOPC (triangle), DOPE (circle), and the bulk solution as a control (diamond).

$$
N_{\mathrm{v}} / N_{\mathrm{s}}=R / \alpha
$$

where $\alpha$ is an undetermined multiplier. Here, we assume fast equilibration on the partition. From homogeneous distribution of GFP within the droplet as noted in Figure 1, the production rate of GFP in the droplet is computed from a volume integral of $V_{\mathrm{GFP}}$, i.e., the product of total volume of the droplet, $4 \pi R^{3} / 3$. Since we assumed a $0^{\text {th }}$-order reaction, the production rate is also expressed by the sum of two different kinetics related to the inner volume $a$, and the membrane surface $b$, as follow

$$
\left(4 \pi R^{3} / 3\right) \cdot V_{\mathrm{GFP}}=a N_{v}+b N_{s} .
$$

By substituting eqs. (1) and (2) into eq. (3), we can obtain the following relation,

$$
\left(4 \pi R^{3} / 3\right) \cdot V_{\mathrm{GFP}}=\{(a(R / \alpha)+b) /(1+R / \alpha)\} \cdot N_{0} .
$$

When the production rate on the membrane surface is more dominant than that in the inner volume, (i.e. $b \gg a)$ and the volume is conserved (i.e., $4 \pi R^{3} / 3=$ constant), the GFP production rate per unit volume, $V_{\mathrm{GFP}}$, can be expressed as

$$
V_{\mathrm{GFP}} \propto\{b /(1+R / \alpha)\} \cdot N_{0} .
$$

When the ratio $R / \alpha$ is larger than $1(R / \alpha \gg 1)$, we can obtain the relation $V_{\mathrm{GFP}} \propto 1 / R$. Homogeneous distribution of GFP within the 
droplet (Figure 1) supports this assumption. Furthermore, the number of substrates $N$ is also proportional to $1 / R$ from the linear relationship between $V_{\mathrm{GFP}}$ and $N$ in eq. (3). In the case of droplets with large $R$ in eq. (4), $V_{\mathrm{GFP}}$ is only determined by the rate of production in the bulk, $a$, which also corresponds to the experimental result shown in Figure 3B. Thus, we can theoretically explain the dependence of the GFP concentration $C_{\mathrm{GFP}}$ and the production rate $V_{\mathrm{GFP}}$ on $1 / R$, under the above-mentioned assumptions including the conditions of $b \gg a$.

The present study shows that protein expression is accelerated in a smaller confinement space, due to the membrane surface. Detailed molecular mechanism of the acceleration is not clear at present, because more than 100 factors organize the translational system ${ }^{20,21}$. Nevertheless, this result reminds us the importance of characteristic cellular size. Additionally, it can help us to realize the importance of membrane structures in cells. Organelles, such as mitochondria or the Golgi apparatus, increase the surface area by stacking membranebound structures. These are understood to hold a large number of membrane proteins ${ }^{22}$. Our results provide another reason for this increased surface area, i.e., the facilitation of protein expression on the membrane surface.

With regard to the lipid-dependence of GFP expression, we focus on the membrane surface potential under the presence of multivalent cations. PG is an anionic lipid and PC is electrically neutral. Although a PE membrane is slightly negative in water, the membrane potential becomes positive in the presence of divalent cations ${ }^{13,23,24}$. Therefore, the order of the lipid-dependence $(\mathrm{PG}>\mathrm{PC}>\mathrm{PE}$ ) might originate from the membrane surface potential under physiological conditions: $\mathrm{PE}$ (positive) $>$ PC (neutral) $>$ PG (negative).

Furthermore, it is known that the cellular membrane has an asymmetric composition between the outer and inner leaflets ${ }^{25}$. It is known that, in many living cells including mammalian cells, PG and PE are found mainly in the inner leaflet, and PC is in the outer leaflet $^{26}$. The precise composition also varies among the organs ${ }^{25}$. Living things may tune this lipid composition to control the gene expression system desirable in each organ.

We have reported a remarkable acceleration of GFP gene expression in a cell-sized droplet coated with a lipid layer. The GFP concentration per unit volume and the production rate were both inversely proportional to the radius of the droplets. The degree of the sizedependence differed among lipid species that comprised the membrane surface, and fell in the following order: PG $>$ PC $>$ PE. These results demonstrate that gene expression in a cell-sized droplet is accelerated in a cell-sized system due to the surrounding membrane surface. In this study, we showed marked acceleration of GFP gene expression by adapting Rabbit reticulocyte lysate as a cell-free translation system. Further experiments with various kinds of expression systems would have of scientific significance toward a further understanding on the underlying physic-chemical propertied on the cellsized confinement.

\section{Methods}

Preparation of droplets encapsulating a GFP gene expression system. The waterin-oil $(\mathrm{W} / \mathrm{O})$ droplet coated by a lipid layer was prepared as described previously ${ }^{13,16}$. Negatively charged lipid, 1,2-dioleoyl-sn-glycero-3-phosphatidylglycerol (DOPG), and zwitterionic lipids, 1,2-dioleoyl-sn-glycero-3-phosphatidylcholine (DOPC) and 1,2-dioleoyl-sn-glycero-3-phosphatidylethanolamine (DOPE), were purchased from Wako Pure Chemical Industries (Osaka, Japan). A dry film of lipids was made on the bottom of a glass tube. Mineral oil (Nacalai Tesque, Kyoto, Japan) was added to the lipid film prior to sonication for $\sim 60 \mathrm{~min}$ at $50^{\circ} \mathrm{C}$. To avoid oxidation ${ }^{27,28}$, the mineral oil was held under an atmosphere of nitrogen gas before preparation. The final lipid concentration in the oil was $0.5-1 \mathrm{mM}$. This procedure resulted in dispersed lipids in oil. To obtain W/O droplets encapsulating a GFP gene expression system, a 5-10 vol $\%$ aqueous solution of the GFP gene expression system was added to the lipid/oil solution, and emulsification was performed by pipetting. As a GFP gene expression system, we used $40 \mu \mathrm{l}$ of TNT rabbit coupled reticulocyte lysate system (Promega, Madison, WI), which included $1 \mu$ g plasmid encoding GFP (more than 200 molecules per $1 \mathrm{pl}$ ) (pCMV6-AC-GFP; Origene, Rockville, MD). GFP expression was performed at the optimal condition in reference to the experimental manual available from the manufacture. It is expected that diffusion of GFP $\left(40 \mu \mathrm{m}^{2} / \mathrm{sec}^{29}\right)$ from surface to inner volume of droplet is much faster than the reaction (lower than 30 times per sec in $1 \mathrm{pL}$ volume). The radius of the resulting $\mathrm{W} / \mathrm{O}$ droplets ranged from 5 to $200 \mu \mathrm{m}$

Microscopic observation. Bright-field and fluorescence microscopy images were obtained with an inverted confocal laser scanning microscope (LSM510; Carl Zeiss, Jena, Germany). GFP was excited with an argon laser $(488 \mathrm{~nm})$ and images were obtained through a $505 \mathrm{~nm}$ long-pass filter (Chroma, Rockingham, VT). All images show equatorial sections of the droplets. The physical size of pinhole aperture was fixed at $\sim 20 \mu \mathrm{m}$ to obtain an intensity that was high enough for analyses. The GFP intensity per unit volume, $I_{\mathrm{GFP}}^{v}$, was obtained from the center of the cross-section image showing droplets with a relatively large radius, $R>10 \mu \mathrm{m}$, to avoid artifacts due to curvature effects. As a control data, $I_{\text {GFP }}^{v}$ of GFP already expressed in the bulk solution was also monitored under the encapsulated in PC droplet. To avoid effects due to the membrane surface, the value of the bulk solution was calibrated at each time point within $\sim 30 \mathrm{~min}$ after the encapsulation. We refer to the $I_{\mathrm{GFp}}^{v}$ normalized by the control data at $3 \mathrm{~h}$ as the GFP concentration $C_{\mathrm{GFP}}$ (r. c., relative concentration). The time-course of $C_{\mathrm{GFP}}$ in the droplets was continuously monitored beginning soon after encapsulation for $\sim 45 \mathrm{~h}$ at $30^{\circ} \mathrm{C}$. We then analyzed the temporal differentiation of $C_{\mathrm{GFP}}$ and obtained the production rate $V_{\mathrm{GFP}}\left(=d C_{\mathrm{GFP}} / d t\right)$.

1. Nomura, S. et al. Gene expression within cell-sized lipid vesicles. Chembiochem 4 , 1172-1175 (2003).

2. Noireaux, V. \& Libchaber, A. A vesicle bioreactor as a step toward an artificial cell assembly. Proc. Natl. Acad. Sci. USA 101, 17669-17674 (2004).

3. Saito, H. et al. Time-Resolved Tracking of a Minimum Gene Expression System Reconstituted in Giant Liposomes. Chembiochem 10, 1640-1643 (2009).

4. Oberholzer, T., Nierhaus, K. H. \& Luisi, P. L. Protein expression in liposomes. Biochem. Biophys. Res. Commun. 261, 238-241 (1999).

5. Yu, W. et al. Synthesis of functional protein in liposome. J. Biosci. Bioeng. 92, 590593 (2001).

6. Ishikawa, K., Sato, K., Shima, Y., Urabe, I. \& Yomo, T. Expression of a cascading genetic network within liposomes. FEBS Lett. 576, 387-390 (2004).

7. Sunami, T. et al. Femtoliter compartment in liposomes for in vitro selection of proteins. Anal. Biochem. 357, 128-136 (2006).

8. Murtas, G., Kuruma, Y., Bianchini, P., Diaspro, A. \& Luisi, P. L. Protein synthesis in liposomes with a minimal set of enzymes. Biochem. Biophys. Res. Commun. 363, 12-17 (2007).

9. Kuruma, Y., Stano, P., Ueda, T. \& Luisi, P. L. A synthetic biology approach to the construction of membrane proteins in semi-synthetic minimal cells. Biochim. Biophys. Acta 1788, 567-574 (2009).

10. Pereira de Souza, T., Stano, P. \& Luisi, P. L. The minimal size of liposome-based model cells brings about a remarkably enhanced entrapment and protein synthesis. Chembiochem 10, 1056-1063 (2009).

11. Bui, H. T. et al. Liposome membrane itself can affect gene expression in the Escherichia coli cell-free translation system. Langmuir 24, 10537-10542 (2008)

12. Hase, M. \& Yoshikawa, K. Structural transition of actin filament in a cell-sized water droplet with a phospholipid membrane. J. Chem. Phys. 124, 104903 (2006).

13. Kato, A., Shindo, E., Sakaue, T., Tsuji, A. \& Yoshikawa, K. Conformational transition of giant DNA in a confined space surrounded by a phospholipid membrane. Biophys. J. 97, 1678-1686 (2009).

14. Tsuji, A. \& Yoshikawa, K. ON-OFF switching of transcriptional activity of large DNA through a conformational transition in cooperation with phospholipid membrane. J. Am. Chem. Soc. 132, 12464-12471 (2010).

15. Hamada, T. et al. Biomimetic Microdroplet Membrane Interface: Detection of the Lateral Localization of Amyloid Beta Peptides. J. Phys. Chem. Lett. 1, 170-173 (2010)

16. Yanagisawa, M., Iwamoto, M., Kato, A., Yoshikawa, K. \& Oiki, S. Oriented Reconstitution of a Membrane Protein in a Giant Unilamellar Vesicle: Experimental Verification with the Potassium Channel KcsA. J. Am. Chem. Soc 133, 11774-11779 (2011)

17. Walde, P., Cosentino, K., Engel, H. \& Stano, P. Giant vesicles: preparations and applications. Chembiochem 11, 848-865 (2010).

18. Pietrini, A. V. \& Luisi, P. L. Cell-free protein synthesis through solubilisate exchange in water/oil emulsion compartments. Chembiochem 5, 1055-1062 (2004).

19. Fiordemondo, D. \& Stano, P. Lecithin-based water-in-oil compartments as dividing Bioreactors. Chembiochem 8, 1965-1973 (2007).

20. Shimizu, Y. et al. Cell-free translation reconstituted with purified components. Nature Biotechnology 19, 751-755 (2001).

21. Schmeing, T. \& Ramakrishnan, V. What recent ribosome structures have revealed about the mechanism of translation. Nature 461, 1234-1242 (2009).

22. Sprong, H., van der Sluijs, P. \& van Meer, G. How proteins move lipids and lipids move proteins. Nat. Rev. Mol. Cell Biol. 2, 504-513 (2001).

23. Egawa, H. \& Furusawa, K. Liposome adhesion on mica surface studied by atomic force microscopy. Langmuir 15, 1660-1666 (1999).

24. Kato, A. et al. Phase Separation on a Phospholipid Membrane Inducing a Characteristic Localization of DNA Accompanied by Its Structural Transition. J. Phys. Chem. Lett. 1, 3391-3395 (2010).

25. van Meer, G., Voelker, D. R. \& Feigenson, G. W. Membrane lipids: where they are and how they behave. Nat. Rev. Mol. Cell Biol. 9, 112-124 (2008). 
26. Devaux, P. F. \& Zachowski, A. Maintenance and Consequences of Membrane Phospholipid Asymmetry. Chem. Phys. Lip. 73, 107-120 (1994).

27. Inouye, S. \& Tsuji, F. I. Evidence for redox forms of the Aequorea green fluorescent protein. FEBS Lett. 351, 211-214 (1994).

28. Heim, R., Cubitt, A. B. \& Tsien, R. Y. Improved green fluorescence. Nature 373 663-664 (1995).

29. Yokoe, H. \& Meyer, T. Spatial dynamics of GFP-tagged proteins investigated by local fluorescence enhancement. Nature Biotechnology 14, 1252-1256 (1996).

\section{Acknowledgement}

We thank Akihiko Tsuji, Shinichiro M. Nomura, Masahiro Takinoue, Masatoshi Ichikawa, Soichiro Kimura, and Yasunori Morimoto for their useful discussions. This work was supported by the Japan Society for the Promotion of Science (JSPS) (Grant-in-Aid for Scientific Research (A); No. 23240044) and by the Ministry of Education, Culture, Sports, Science and Technology (Scientific Research on Innovative Areas; No. 23106712).

\section{Author contributions}

$\mathrm{MY}, \mathrm{KF}$ and $\mathrm{KY}$ wrote the manuscript. AK, YS and KY designed research. AK, MY, YS and KF performed experiments. AK, MY, KF and KY analyzed data. All authors reviewed the manuscript.

\section{Additional information}

Supplementary information accompanies this paper at http://www.nature.com/ scientificreports

Competing financial interests: The authors declare no competing financial interests. License: This work is licensed under a Creative Commons

Attribution-NonCommercial-ShareAlike 3.0 Unported License. To view a copy of this license, visit http://creativecommons.org/licenses/by-nc-sa/3.0/

How to cite this article: Kato, A., Yanagisawa, M., Sato, Y.T., Fujiwara, K. \& Yoshikawa, K. Cell-Sized confinement in microspheres accelerate the reaction of gene expression. Sci. Rep. 2, 283; DOI:10.1038/srep00283 (2012) 